\title{
Immunohistochemical and biochemical assay of versican in human sound predentine/dentine matrix
}

\author{
A. Ruggeri,, ${ }^{1}$ G. Orsini, ${ }^{2}$ A. Mazzoni, ${ }^{1}$ F. Nato, ${ }^{1,3}$ V. Papa, ${ }^{1}$ M. Piccirilli, ${ }^{4}$ A. Putignano, ${ }^{2}$ G. Mazzotti, ${ }^{1}$ \\ E. De Stefano Dorigo, ${ }^{5}$ L. Breschi ${ }^{5,6}$
}

${ }^{1}$ Department of SAU\&FAL, University of Bologna, Italy; ${ }^{2}$ Department of Clinical Sciences and

Stomatology, Polytechnic University of Marche, Ancona, Italy; ${ }^{3}$ Department of SUAN, University of Urbino, Urbino Italy; ${ }^{4}$ Department of Stomatology and Oral Science, University of Chieti-Pescara, Italy; ${ }^{5}$ Unit of Dental Sciences and Biomaterials, Department of Biomedicine, University of Trieste; ${ }^{\circ}$ GMM-CNR, Unit of Bologna c/o IOR, Bologna, Italy

(C)2009 European Journal of Histochemistry

Aim of this study was to investigate the distribution of versican proteoglycan within the human dentine organic matrix by means of a correlative immunohistochemical analysis with field emission in-lens scanning electron microscope (FEI-SEM), transmission electron microscope (TEM), fluorescence microscope (FM) and biochemical assay. Specimens containing dentine and predentine were obtained from non carious human teeth and divided in three groups: 1) FEI-SEM group: sections were exposed to a pre-embedding immunohistochemical procedure; 2) TEM group: specimens were fixed, demineralised, embedded and submitted to a post-embedding immunohistochemical procedure; 3) FM group: sections mineralised and submitted to a pre-embedding immunohistochemical procedure with fluorescence labelling. Specimens were exposed to two different antibodies to assay distribution of versican fragments and whole versican molecule. Western Blotting analysis of dentine and pulp extracts was also performed. The correlative FEI-SEM, TEM and FM analysis revealed positive immunoreaction for versican fragments both in predentine and dentine, while few gold particles identifying the whole versican molecule were found in predentine only under TEM. No labelling of versican whole molecule was detected by FEI-SEM and FM analysis. The immunoblotting analysis confirmed the morphological findings. This study suggests that in fully developed human teeth versican fragments are significant constituents of the human dentine and predentine organic matrix, while versican whole molecule can be visualised in scarce amount within predentine only. The role of versican fragments within human dentine organic matrix should be further elucidated.

Key words: versican, dentine matrix, immunohistochemistry, TEM, FEISEM, fluorescence microscope.

Correspondence: Lorenzo Breschi,

Division of Dental Sciences and Biomaterials,

Department of Biomedicine, University of Trieste,

Via Stuparich, 1, 34129 Trieste, Italy

Tel:. +39.040.3992192.

Fax: +39.040.3992665.

E-mail: Ibreschi@units.it

Paper accepted on 5 June 2009

European Journal of Histochemistry

2009; vol. 53 issue 3 (July-September): 125-134
The human dentine organic matrix is composed by a large complex of macromolecules capable of self-assembly. The dentine matrix is represented predominantly by type I collagen and completed by non collagenous glycoproteins, elastin, hyaluronan and proteoglycans (PGs). While type I collagen is the backbone of the dentine with a predominant structural role, non-collagenous proteins, and in particular PGs, are believed to play fundamental functional roles during odontogenesis, mineralization and homeostasis of dentine.

The process of odontogenesis appears to be controlled by a precise sequential expression of a pool of extracellular non-collagenous proteins that induces modifications within the extracellular environment of the predentine leading to the formation of the dentine matrix (Embery et al., 2001). Similarly, dentine mineralization involves a dynamic transition from the unmineralised predentine to the mineralised mature dentine, in which the role of specific regulative mineralisation proteins appears to be pivotal in the precipitation of the minerals and in the formation of apatite crystals (Embery et al., 2001). In particular, PGs has been shown to play crucial role in the mineralisation processes of dentine (Embery et al., 2001; Waddington et al., 2003).

PGs are macro-molecules where, at least, one glycosaminoglycan side chain (GAGs) is covalently attached to the protein core of the molecules. Their size and structure can change and can be differentially found intracellulary, on the cell surface, or within the extracellular matrix. The majority of PGs have been identified by their antigenic and structural properties suggesting numerous biological functions (Embery et al., 2001). Biochemical, histochemical and immunohistochemical studies on PGs of dentine and predentine have yielded sufficient information to indicate that the predominant PGS 
belong to the small leucine-rich interstitial family (SLRP) (Fisher et al., 1983; Yoshiba et al., 1996). They include decorin and biglycan (Waddington et al., 2003; Orsini et al., 2007), which bear one or two chondroitin/dermatan sulphate GAGs, lumican, fibromodulin and osteoadherin that bear keratan sulphate GAGs chains (Iozzo et al., 1997, 1999; Neame et al., 2000). A second pool of PGs belongs to the large aggregation chondroitin/keratan sulphate family named hyaluronan-binding (HA), including aggrecan, versican, brevican and neurocan (Yamauchi et al., 1997).

Versican was firstly isolated in chicken mesenchymal tissue, and it has been found to be expressed also in keratinocytes, smooth muscle cells of the vessels, brain and mesangial cells of the kidney. Similar PGs have been found in other connective tissues (Zimmermann et al., 1989; Shinomura et al., 1990; Zimmermann et al., 1994; Landolt et al.,1995) and recent studies have shown that, within the dental tissues, versican has been localised in gingival fibroblasts culture, dental pulp complex (Yamauchi et al., 1997; Bartold et al., 1995; Shibata et al., 2000; Shibata et al., 2002; Robey et al., 1993; Ababneh et al., 1999; Cheng et al., 1999), dentine (Waddington et al., 2003), cementum (Ababneh et al., 1999; Cheng et al., 1999) and periodontal ligament (Sato et al., 2002).

Within the dentine organic matrix versican can be detected either as fragments or as whole molecule. Waddington et al. (2003) reported that versican is mainly present as its degradation products (fragments), whereas the whole molecule has been isolated by Shibata et al. $(1999 ; 2000)$ in rat dental pulp tissue.

The aim of this study was to localise versican $P G$ in human mature dentine by an immunohistochemical technique using a monoclonal antibody antiversican (towards the whole molecule) and a polyclonal antibody anti-versican fragments, under high resolution field emission in-lens scanning electron microscope (FEI-SEM), electron transmission microscope (TEM) and fluorescence microscope (FM) and to confirm the morphological findings by a biochemical assay.

\section{Materials and Methods}

All reagents were purchased by Sigma Chemical Co (St. Louis, USA, ) if not differently specified.
Fifteen human sound molars scheduled for extraction were selected for the study. Patients with a mean age of 28.7 years enrolled in this protocol provided informed consent form, which has been approved by the Ethic Committee of the University of Bologna. Roots of the extracted teeth were immediately removed and the crown portions were transversally sectioned, using a low speed diamond saw (Remet, Casalecchio di Reno, Italy) under water irrigation. One mm-thick dentine disks $(\mathrm{N}=30)$ were obtained by middle/deep dentine and polished by increasing grid SiC paper under constant deionised water irrigation. Specimens were then ultrasonically cleaned for $3 \mathrm{~min}$ in $0.05 \mathrm{M}$ Tris $\mathrm{HCl}$ buffer solution (TBS) at $\mathrm{pH}$ 7.6. Specimens were then equally and randomly assigned to the following treatment groups $(\mathrm{N}=10)$ : 1) FEI-SEM group: un-fixed demineralised specimens were processed for a pre-embedding immunohistochemical procedure; 2) TEM group: specimens were immediately fixed, decalcified and processed for post-embedding immunohistochemistry; 3) FM group: un-fixed and un-demineralised specimens were submitted to a pre-embedding immunohistochemical technique followed by corresponding fluorochrome-conjugated.

\section{Pre-embedding technique - tissue processing for the FEI-SEM group}

Un-fixed specimens of the FEI-SEM group were processed for a pre-embedding immunolabelling procedure in accordance with Breschi et al. (2002). Dentine samples were etched with 10\% citric acid for 15 seconds to remove the smear layer and to expose dentine surface and immunolabelled using either a rabbit polyclonal primary antibody (IgG anti-versican LF-99, generously donated by Dr L. Fisher, National Institutes of Health, NIDR, Bethesda, MD, USA) in order to reveal the presence of versican fragments within dentine matrix (Waddington et al., 2004), or a mouse monoclonal primary antibody (IgG $12 \mathrm{C} 5$ anti-Versican HAbinding region, Developmental Studies Hybridoma Bank, Iowa City, IA, USA) in order to identify versican whole protein (Shibata et al., 1999, 2000).

In brief, specimens were immersed in $0.05 \mathrm{M}$ Tris $\mathrm{HCl}$ buffer solution (TBS) at $\mathrm{pH} 7.6$ with $0.15 \mathrm{M}$ $\mathrm{NaCl}$ and $0.1 \%$ bovine serum albumin and preincubated for $30 \mathrm{~min}$ in TBS $0.05 \mathrm{M}$ containing normal goat serum (British BioCell International, Cardiff; UK) at $\mathrm{pH}$ 7.6. Overnight incubation was 
performed using either one of the primary antibodies at $4^{\circ} \mathrm{C}$. After incubation, dentine specimens were rinsed with TBS $0.05 \mathrm{M}$ at $\mathrm{pH} 7.6$ and TBS $0.02 \mathrm{M}$ at $\mathrm{pH}$ 8.2. Gold labelling was performed using secondary antibody conjugated with $15 \mathrm{~nm}$ gold particles (IgG goat anti-rabbit IgG for LF-99 analysis or IgG goat anti-mouse IgG for $12 \mathrm{C} 5$ visualisation, British BioCell International). Secondary antibodies were diluted in $0.02 \mathrm{M}$ TBS at $\mathrm{pH} 8.2(0.02 \mathrm{M}$ Tris $\mathrm{HCl}$ buffered at $\mathrm{pH} 8.2$ with $0.15 \mathrm{M} \mathrm{NaCl}$ and $0.1 \%$ BSA) for $90 \mathrm{~min}$ at room temperature. Specimens were then rinsed in TBS $0.02 \mathrm{M}$ at $\mathrm{pH}$ 8.2 and in deionised water. Specimens were then fixed in $2.5 \%$ glutaraldehyde in $0.1 \mathrm{M}$ cacodylate buffer at $\mathrm{pH} 7.2$ for 4 hours, rinsed in $0.2 \mathrm{M}$ cacodylate buffer, dehydrated in ascending ethanol series, air dried from hexamethyldisilazane (HMDS) and coating with carbon graphite (Perdigao et al., 1995). Observations were performed under a FEI-SEM (JEOL JSM 890, JEOL, Tokyo, Japan) at $7 \mathrm{KV}$ accelerating voltage and 1x10-11 Amp probe current. Final images were obtained by mixing with both back scattered (BSI) and secondary electron (SEI) signals.

\section{Post embedding technique: tissue processing for TEM group}

Dentine disks assigned to the TEM group were rinsed in saline solution and immediately fixed in $4 \%$ formaldehyde (made by depolymerising paraformaldehyde)-0.1\% glutaraldehyde in $0.1 \mathrm{M}$ cacodylate buffer at $\mathrm{pH} 7.2$, overnight at $4^{\circ} \mathrm{C}$. Then, specimens were washed for $1 \mathrm{~h}$ in the buffer alone and demineralised using $4.13 \%$ EDTA (Warshawsky and Moore 1967) for approximately three months, followed by extensive washing with $0.1 \mathrm{M}$ cacodylate buffer at $\mathrm{pH}$ 7.2. Samples were then dehydrated in graded concentrations of ethanol and embedded in LR White resin (London Resin, Berkshire, UK). Semi-thin sections ( $1 \mu \mathrm{m}$ ) were cut with glass knives on a Reichert Jung Ultracut E Ultramicrotome (Reichert-Jung, Leica Microsystems, Milan, Italy) and stained with toluidine blue. Selected areas of the $1 \mu \mathrm{m}$ thick sections were trimmed for ultra thin sectioning $(80 \mathrm{~nm})$ using a diamond knife and mounted on 200-mesh formvar carbon-coated nickel grids.

Grid-mounted tissue thin sections were processed for immunohistochemical labelling. The incubations were carried out following the same protocol as previously described in the pre-embedding tech- nique (Breschi et al., 2003) using each one of the two primary antibodies anti-versican previously tested. In particular sections were rinsed and gold labelling was performed using the IgG goat antirabbit IgG for LF-99 distribution analysis and IgG goat anti-mouse IgG for $12 \mathrm{C} 5$ visualisation. Both antibodies were conjugated with $15 \mathrm{~nm}$ colloidal gold. Grids were then stained with $4 \%$ uranyl acetate and lead citrate for examination in a Jeol 1010 TEM operated at $60 \mathrm{kV}$ (JEOL Ltd., Tokyo, Japan). The TEM was connected with a Digital Camera MegaView III equipped with the Analysis Imaging System GmbH (Munster, Germany).

\section{Fluorescence labelling: tissue processing for FM group}

For immunofluorescence labelling, the mineralised dentine disks were left un-fixed and rinsed in $0.1 \mathrm{M}$ sodium cacodylate, at pH 7.2. Then, $100 \mu \mathrm{m}$ sections were prepared using a high-precision cutting machine equipped with diamond disks (Remet) and polished until reaching $50 \mu \mathrm{m}$-thick specimens, that were immediately processed for incubation using one of the primary antibodies, IgG goat antirabbit for LF-99 or IgG goat anti-mouse for $12 \mathrm{C} 5$ distributions. Samples were then incubated for 90 min with secondary antibodies conjugated with fluorescein isothiocynate (FITC) and diluted 1:100 in $0.02 \mathrm{M}$ TBS $\mathrm{pH} 8.2$ at room temperature in dark conditions. An anti-rabbit IgG antibody was used for LF-99 identification, while an anti mouse IgG was applied on anti-12C5 incubated specimens. Specimens were then washed for $10 \mathrm{~min}$ in $0.02 \mathrm{M}$ TBS pH 8.2, briefly rinsed with distilled water, and coverslipped with aqueous mounting medium (Vecta-Shield, Vector Laboratories, Burlingame, CA, USA). Labelled sections were observed under a Nikon Eclipse E-800 (Nikon, Tokyo, Japan).

\section{Controls for immunohistochemical techniques}

Controls were performed in accordance with previous studies (Breschi et al., 2002, 2003; Suppa et al., 2006; Ruggeri et al., 2007; Orsini et al., 2007; Orsini et al., 2007; Mazzoni et al., 2009.), i.e. for the pre-embedding technique: (1) processed as previously described and incubated overnight in TBS $0.05 \mathrm{M}$ at $\mathrm{pH} 7.6$ without the primary antibody; (2) incubated after organic matrix removal, obtained with a water solution of $5 \% \mathrm{NaOCl}$ applied for $5 \mathrm{~min}$ at room temperature onto the dentine surface. 
Controls of the post-embedding technique consisted of dentine specimens processed as previously described and incubated overnight in TBS $0.05 \mathrm{M}$ at $\mathrm{pH} 7.6$ without the primary antibody.

Controls of the FM group consisted on specimens (1) incubated with non-immune serum or (2) with the secondary antibody, only.

\section{Western blot analysis}

Since protein extracts of predentine only cannot be obtained on human teeth due to the impossibility of isolating pure predentine from pulp and dentine, we investigated human pulverised dentine and pulp extracts. For this purpose human teeth were longitudinally cut to expose the pulp tissue and enamel, pulp and predentine were removed using a diamond bur to obtain specimens of mineralised dentine only. Dentine aliquots of approximately 750 mg wet wt were obtained from pulverized dentine, in accordance with Mazzoni et al. (2007).

Dentine specimens were decalcified with $0,5 \% \operatorname{EDTA}(1: 5 \mathrm{w} / \mathrm{v})$ for $24 \mathrm{~h}$ at $4^{\circ} \mathrm{C}$, dentine aliquots were then collected by centrifugation for $10 \mathrm{~min}$ at $4000 \mathrm{rpm}$ at $4^{\circ} \mathrm{C}$ and rinsed in water. Specimens were then incubated with $Q 1$ extraction buffer (Epigentek Group Inc, NY, USA) ( $1: 2 \mathrm{w} / \mathrm{v}$ ) and proteins were extracted by gentle rocking at $4^{\circ} \mathrm{C}$ overnight. Remnants of dentine powder were removed by centrifugation at $14000 \mathrm{rpm}$ for 20 min at $4^{\circ} \mathrm{C}$ then protein supernatants were collected, loaded onto a centrifugal concentrator, heated to $95^{\circ} \mathrm{C}$ for $5 \mathrm{~min}$ and ice-cooled.

Primary cultures and cell lysates were prepared in accordance with Teti et al., (2008). Equal amounts of protein extracts $(75 \mu \mathrm{g})$ were separated on sodium dodecylsulfate-polyacrylamide gel electrophoresis and electrotransferred onto nitrocellulose membranes. Membranes were briefly washed in PBS-0.1\% Tween-20 (PBS-T) and nonspecific binding sites were blocked in $5 \%$ non-fat dry milk in PBS-T for $1 \mathrm{~h}$ at room temperature with gentle agitation. After PBS-T washes, membranes were incubated $3 \mathrm{~h}$ at $37^{\circ} \mathrm{C}$ with primary antibodies diluted according to the manufacturer's instructions. Membranes were then incubated with horseradish peroxidase-conjugated secondary antibodies diluted $1: 1000$ in 5\% non-fat dry milk in PBS-T for $1 \mathrm{~h}$ at room temperature with gentle agitation. Antibody binding was detected by SuperSignal West Pico Chemiluminescent Substrate (GE Healthcare, London, UK).

\section{Results}

Immunoreaction for LF-99, which identifies versican fragments, and for $12 \mathrm{C} 5$, identifying the versican whole molecule were analised in predentine and dentine layers using a correlative approach by means of FEISEM, TEM and FM (Figures $1,2,3$ ). In addition Western Blots analysis confirmed the morphological pattern. The following sections summarize common features that have been shown by the samples of each group.

\section{Pre-embedding technique: FEISEM analysis}

Positive immunoreaction for LF-99 (versican fragments) in the predentine layer was evident due to the presence of electron reflective particles (which appear as white spherical spots) scattered within the organic matrix (Figure 1). Several gold particles were associated with the intricate network of collagen fibrils bundles (Figure 1a), while a decrease of labelling for the same antibody was observed in dentine (Figure 1c), in which versican fragments appear as distributed within the organic matrix, mainly localised in the peritubular dentine regions. On the other hand, the immunoreaction for versican whole molecule (12C5) was very weak in predentine only (Figure $2 \mathrm{a}$ ) and almost absent in dentine (Figure 2c). Control specimens showed no labelling, thus confirming the specificity of the primary antibodies (data not shown).

\section{Post-embedding technique: TEM analysis}

Correlative TEM findings for LF-99 were obtained in human predentine and dentine. Indeed, a moderately intense labelling was associated with the collagen fibrils within the thickness of predentine layer (Figure $1 b$ ), while a moderate to weak labelling was present in dentine (Figure 1d), without any evident gradient among the different dentine region; both peritubular and intertubular dentine than dentinal tubules presented only scattered gold particles.

The immunoreaction for $12 \mathrm{C} 5$ antibody was generally lower than the one observed for LF-99, both in predentine (Figure $2 b$ ) and dentine (Figure $2 d$ ). In particular, although dentine showed some positive labelling for versican whole molecule, the intact molecule was very scarce within the predentine. In controls of TEM preparations, only few randomly distributed gold particles were seen over the tissue sections (data not shown). 


\section{Antibody LF-99}
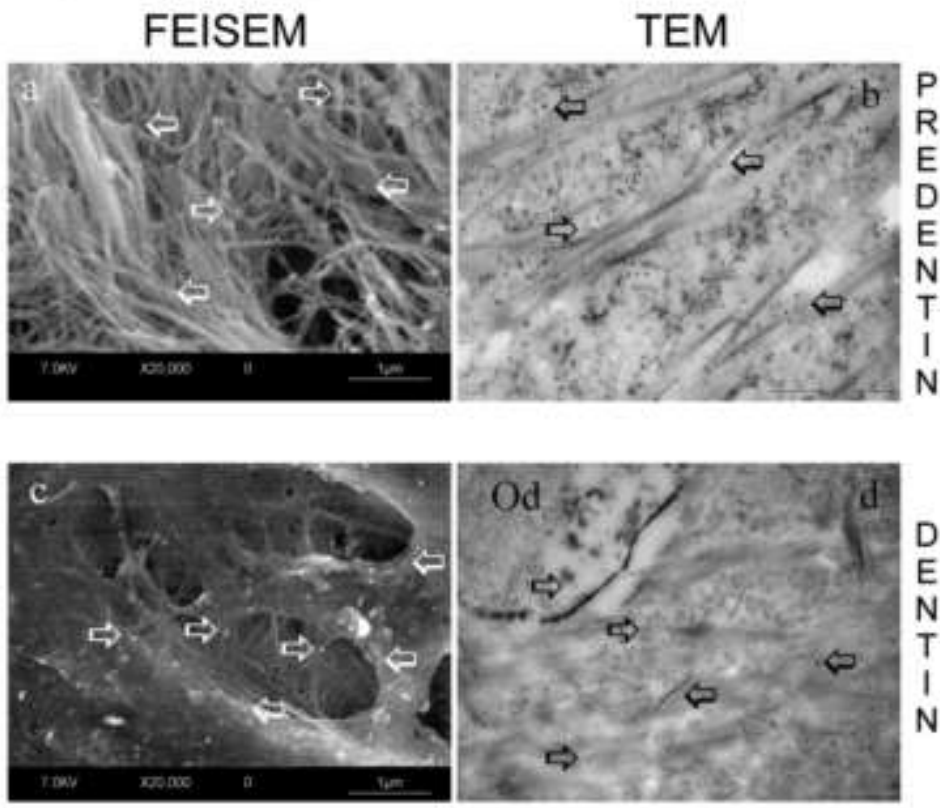

Antibody $12 \mathrm{C} 5$
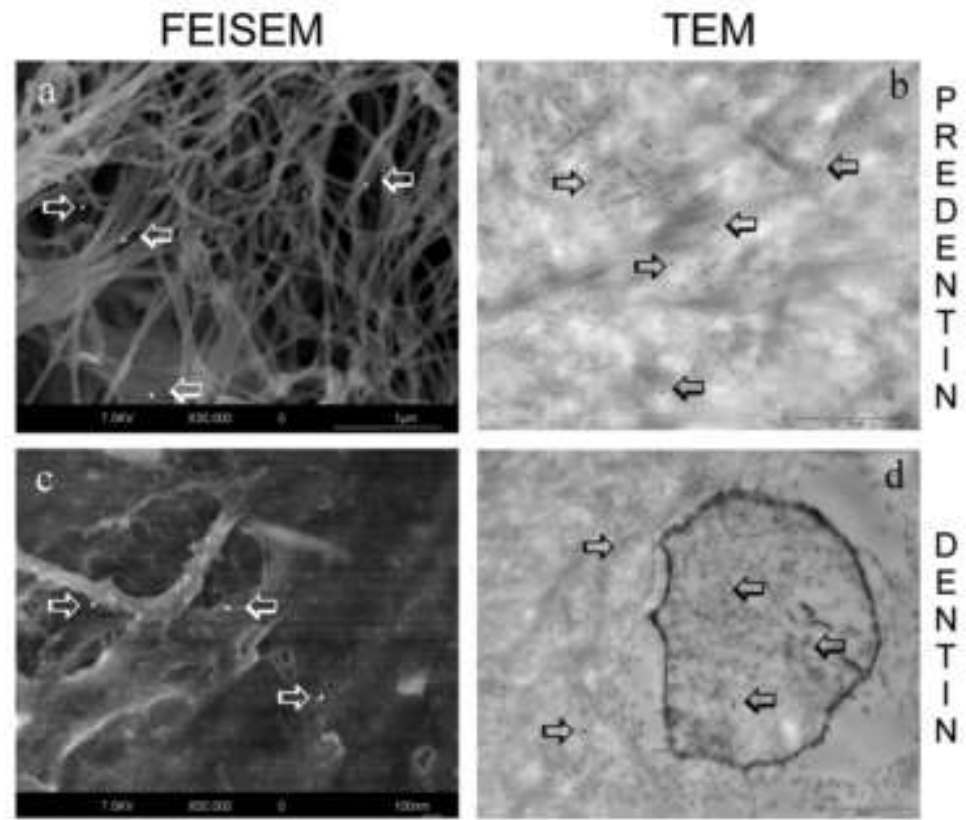

Figure 1. Micrographs of human dentine preparations obtained by means of FEISEM and TEM, revealing the immunoreaction for LF-99 polyclonal antibody, which identifies versican fragments. (a) FEISEM predentine micrograph revealing an intricate network of collagen fibril bundles moderately labeled for LF-99 (arrows). (b) TEM image showing gold nanoparticles (arrows) in the predentine layer: a diffuse and moderately intense labeling can be seen over the collagen fibrils (Bar: $10 \mu \mathrm{m})$. (c) FEISEM image of the dentine revealing a generally weak labeling mainly distributed over the peritubular zone (arrows). (d) TEM image of the dentine matrix revealing a moderate to weak labeling over peritubular and intertubular regions; Od: odontoblast process (Bar: $10 \mu \mathrm{m}$ ).
Figure 2. FEISEM and TEM micrographs of human dentine showing immunoreactivity for versican whole molecule by using $12 \mathrm{C} 5$ monoclonal antibody. (a) FEISEM micrograph of the predentine zone revealing a very weak reaction for versican whole molecule (arrows) over the collagen fibrils. (b) TEM image revealing several randomly dispersed gold nanoparticles (arrows) over the predentine layer sometimes associated with the collagen fibrils (Bar: $10 \mu \mathrm{m})$. (c, d) In both images a few randomly scattered gold particles were detected within the dentine matrix, being almost absent in the FEISEM micrograph, while slightly more numerous in the TEM micrograph (Bar: 10 $\mu \mathrm{m})$, mainly localised over the intertubular dentine and also present over the odontoblast process (arrows).

\section{Fluorescence labelling technique: FM analysis}

The immunofluorescence analysis showed distribution of LF-99 primary antibody (i.e. anti-versican fragments) in both predentine and dentine tissues (Figure 3a). On the contrary, specimens incubated with anti-12C5 (i.e. identifying the whole versican molecule) showed some positive immunos- taining mainly within the predentine layer (Figure 3b) confirming the correlative data obtained with FEI-SEM and TEM analysis.

Control specimens incubated with nonimmune serum or secondary antibody only exhibited some background fluorescence but no significant labelling over the tissue sections (Figure $3 c$ ). 


\section{FM Images}
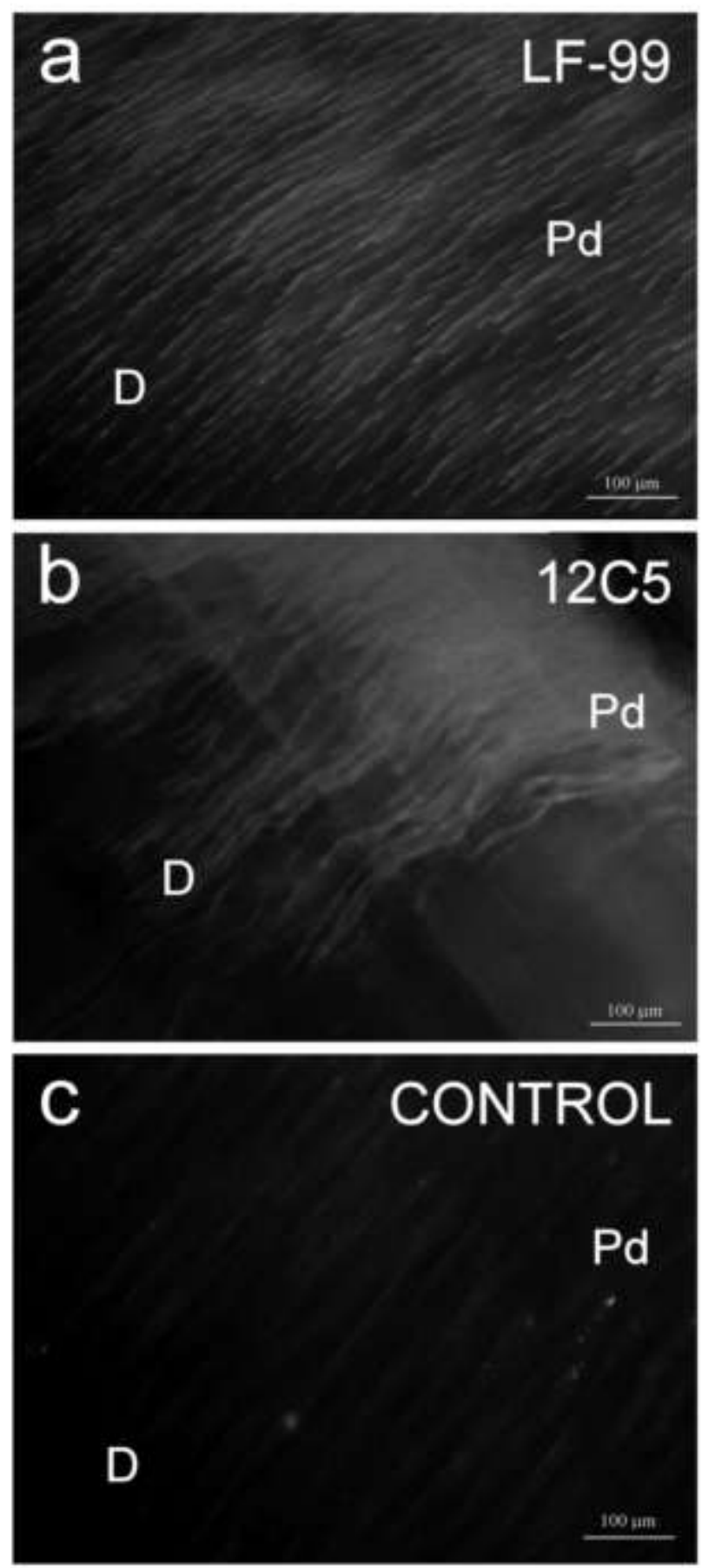

Figure 3. FM micrographs of human predentine and dentine showing fluorescence immunoreactivity for versican by using both antibodies. (a) FM micrograph reveals a positive activity in both portions of the predentine (Pd) and dentine (D) layers; bar $=100 \mu \mathrm{m}$. (b) FM image revealing positive fluorescence immunostaining in particular in predentine (Pd) layer, typically of 12C5 antibody recognising molecula of versican proteoglycan, while fluorescence decrease towards dentine tissue (D); bar $=100 \mu \mathrm{m}$. (c) Control FM micrograph of human predentine and dentine, with no positive reaction; bar $=100 \mu \mathrm{m}$.

\section{Western blot analysis}

Western blot analysis confirmed the morphological data as presence of versican whole molecule and fragments were revealed in pulp extracts (Figure 4, lane 2), while versican fragments only were found within demineralised dentine (Figure 4, lane 1). In particular, Western blot analysis with LF-99 antibody demonstrated the presence of the versican fragments in demineralised dentine, while only a faint diffuse staining was found for anti12C5 antibody. Conversely, Western Blot analysis for pulp extracts was found to be positive with both antibodies (LF-99 and 12C5; Figure 4).

\section{Discussion}

Versican has been proposed to possibly act as a temporary scaffold in the capture of space during the initial formation of extracellular matrix (Robey et al., 1997) and during processes such as tooth eruption and early dentinogenesis (Waddinghton et al., 2003; Sone et al., 2005). In fact, versican is a member of an important pool of PGs that led to the initial formation of the extracellular matrix (Zimmermann et al., 1989) by capturing a temporal space for succeeding tissues/cells embryonic (Shinomura et al., 1990; Landolt et al., 1995). Despite versican localisation as been demonstrated in dental pulp (Shibata et al. 2000, 2002; Robey et al., 1993), periodontal ligament (Sato et al., 2002; Shibata et al., 1999), dentine (Waddinghton et al., 2003) and cementum (Ababneh et al., 1999; Cheng et al., 1999), its function and precise distribution within the sound dentine are still unclear.

The present study revealed that versican main-

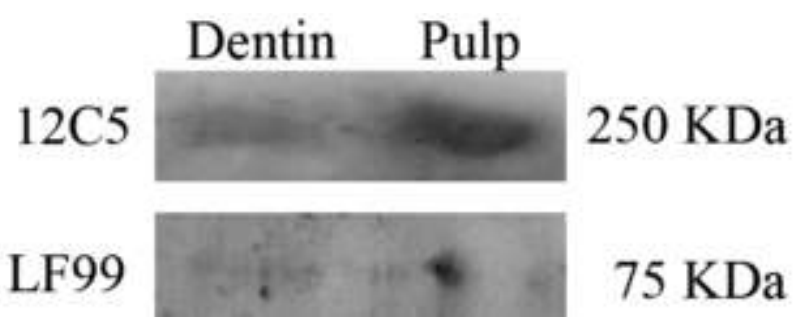

Figure 4. Western Blot analysis of the human EDTA-decalcified pulverized dentine and human pulp fibroblasts. Positive reaction was found for LF99 antibody within the dentine extracts (lane 1), while only a faint staining was detectable for the $12 \mathrm{C5}$ antibody. Conversely, pulp extracts revealed positive immunoreactions for both tested antibodies, thus demonstrating presence of fragments and whole versican proteoglycan molecules (lane 2). 
tained its antigen integrity after citric acid etching and, using an immuno-gold labelling protocol (Breschi et al., 2003), it was possible to visualise at high resolution the three-dimensional distribution of versican within dentine and predentine as well as its relationship with the collagen fibrils. Interestingly, the results of the study confirmed that versican fragments (identified using LF-99 antibody) are present within the intertubular dentine and homogenously distributed in the predentine matrix, while the whole molecule of versican (antibody $12 \mathrm{C5}$ ) can be identified in the predentine only. Western Blots confirmed the immunohistochemical findings.

By means of the pre-embedding immunohistochemical protocol and using the high-resolution SEM, dentine morphology and versican labelling patterns were simultaneously analysed on the same specimen (Breschi et al., 2003) (Figure 1). In this case, labelling appeared as electron reflecting particles, i.e. white spots of approximately $15 \mathrm{~nm}$ in diameter, whereas using TEM, gold labelling was identified as electron-dense particles (Figure 2), i.e. black spots. TEM labelling (post-embedding technique) is referred to protein epitopes, exposed by the ultramicrotome diamond blade, while FEISEM and FM labelling (pre-embedding technique) is referred to epitopes present on the surface of the dentine matrix exposed by mineral removal obtained with citric acid. The different pre-embedding vs post-embedding approach leads to different labelling probably due to the different access to the epitopes.

In a previous study, Waddington et al., (2003) have simultaneously analised the three PGs decorin, biglycan and versican, biochemically identifying them in the dentinal fractions of calves teeth. Decorin and biglycan can regulate the influence of the mineralisation process in collagen stabilisation, collagen fibrillogenesis, calcium binding potential, interaction with hydroxyapatite, and inhibition of crystal growth (Embery et al., 2001; Orsini et al., 2007; Septier et al., 2002; Waddington et al., 2002). On the other hand, large PGs mainly synthesised within the predentine matrix, such as versican, play important roles in matrix formation and in prevention of premature mineralisation (Waddinghton et al., 2003). Indeed, versican (molecular weight of 1 million $\mathrm{kDa}$ with a protein core of $360 \mathrm{KDa}, 12$ chondroitin sulphate (chains of $45 \mathrm{KDa}$ ) is present in early stages of dentine formation and, by association with hyaluronan, demarcates space that will ultimately become mineralised (Robey et al., 1996). Afterwards, it is subsequently degraded, releasing fragments that are homologous to the extracellular matrix and become replaced by the two small leucine-rich PGs containing chondroitin sulphate: decorin (with one chondroitin sulphate chain), and biglycan (with two chondroitin sulphate chains). Indeed, the authors (Waddinghton et al., 2003) showed for the first time that LF-99 recognises versican fragments of 65 and $57 \mathrm{KDa}$ in predentine and the $65 \mathrm{KDa}$ protein species and faint diffuse staining of 150/75 $\mathrm{KDa}$ in dentine, thus concluding that versican is not present in predentine and dentine as the intact core protein, while as metabolite products of it. This may explain why a moderate labelling was found using the LF-99 antibody which recognised fragments dispersed in predentine and dentine, while a weak reaction for the $12 \mathrm{C} 5$ antibody (recognising the whole molecule) occurred in predentine and was almost absent in dentine of the examined mature sound teeth (also confirmed by the Western Blot analysis).

Ilic et al. (2005) confirmed that small leucinerich $P G s$ are the most abundant $P G s$ present in organic matrix, whereas large PGs are lower amounts. In the present study it was elucidated that many versican fragments are still present after mineralisation as proteolytic products of its core protein, i.e. they are still present in the sound dentine. This may suggest that the accumulation of $P G s$ fragments is related to a normal turnover of $P G s$ in the extracellular matrix of connective tissues, and that the decrease of versican from predentine to dentine can be correlated to the degradation of products of the versican core molecules.

The potential candidates of the degradation of the extracellular matrix are enzymes involved in the degradation of versican are disintegrins and matrix metalloproteases with thrombospondin type 1 and 4 (ADAMTS-1 and ADAMTS-4). Indeed, ADAMTS, belonging to a family of extracellular proteases, have been implicated in cleaving PGs, such as aggrecan, versican and brevican (Hirohata et al., 2001; Apte et al., 2004). Sone et al. (2005) has recently demonstrated that recombinant forms of these enzymes can cleave various versican isoforms giving rise to different fragments.

The function of these metabolites is unclear, but it is supposed that enzymes of the ADAMTS family 
can cooperate in the remodelling process of PGs like versican, constituting the extracellular environment of teeth which contributes to tooth eruption (Sone et al., 2005). Indeed, the reduced level of versican from predentine to dentine may be related to its proposed functions in dentine matrix assembly and stabilisation. Thus, versican may characterize a more provisional and loosely organised extracellular dentine matrix. Versican probably does not act alone; indeed, the challenge for further studies will be to sort out the multitude of versican interactions, whether they can be direct or indirect, i.e. the functions of versican result in specific and targeted events that eventually cause a change in cell phenotype. For instance, potential indirect interactions may be played by different matrix metalloproteases present in dentine matrix (Mazzoni et al., 2007,2009 ) that in turn may have a central role in degradation of the extracellular matrix such as remodelling dental matrix (Maruya et al., 2003; Tsubota et al., 2002). On the other hand, ADAMTS may play an important role only during mineralisation process of dentine matrix. Further research will be required to understand the involvement of matrix metalloproteases and ADAMTS in formation and remodeling of the dentine matrix.

\section{Acknowledgements}

The authors wish to thank Dr. Larry Fisher (National Institute of Dental and Craniofacial Research, Bethesda, MD, USA) for generously donating IgG anti-versican (LF-99) antibody and $\mathrm{Mr}$ Aurelio Valmori for photographical work. The study was partially supported by grants from "Università di Trieste-Finanziamento Ricerca d'Ateneo" and MIUR, Italy.

\section{References}

Ababneh KT, Hall RC, Embery G. The proteoglycans of human cementum: immunohistochemical localization in healthy, periodontally involved and ageing teeth. J Periodontal Res 1999;34:87-96.

Apte SS. A disintegrin-like and metalloprotease (reprolysin type) with thrombospondin type 1 motifs: the ADAMTS family. Int $J$ Biochem Cell Biol 2004;36:981-5.

Bartold PM, Moule AJ, Li H, Rigby P. Isolation and characterization of the proteoglycans synthesized by adult human pulp fibroblasts in vitro. Int Endo Jour 1995;28:163-71.

Breschi L, Lopes M, Gobbi P, Mazzotti G, Falconi M, Perdigão J. Dentin proteoglycans: an immunocytochemical FEISEM study. $J$ Biomed Mater Res 2002;61:40-6.

Breschi L, Perdigão J, Lopes M M, Gobbi P, Mazzotti G. Morphological study of resin-dentin bonding with TEM and in-lens FESEM. Am J Dent 2003;16:267-74.

Cheng $\mathrm{H}$, Caterson B, Yamauchi M. Identification and immunolocaliza- tion of chondroitin sulfate proteoglycans in tooth cementum. Connect Tissue Res 1999;40:37-47.

Embery G, Hall RC, Waddington RJ, Septier D, Goldberg M. Proteoglycans in dentinogenesis. Crit Rev Oral Biol Med 2001;12:331-49.

Fisher LW, Termine JD, Dejter SW Jr, Whitson SW, Yanagishita M, Kimura JH, Hascall VC, Kleinman HK, Hassell JR, Nilsson B. Proteoglycans of developing bone. J Biol Chem 1983;258:6588-94.

Hirohata S. ADAMTS family-new extracellular matrix degrading enzyme. Seikagaku 2001;73:1333-7.

Ilic MZ, Carter P, Tyndall A, Dudhia J, Handley CJ Proteoglycans and catabolic products of proteoglycans present in ligament. Biochem $\mathrm{J}$ 2005; 385(Pt 2):381-8.

Iozzo RV. The biology of the small leucine-rich proteoglycans: functional network of interactive proteins. J Biol Chem 1999; 274:188436.

Iozzo RV. The family of the small leucine-rich proteoglycans: key regulators of matrix assembly and cellular growth. Crit Rev Biochem Mol Biol 1997;32:141-74.

Landolt RM, Vaughan L, Winterhalter KH, Zimmermann DR. Versican is selectively expressed in embryonic tissues that act as barriers to neural crest cell migration and axon outgrowth. Develo 1995;121:2303-12.

Marks SC Jr, Schroeder HE. Tooth eruption: Theories and facts. Anat Res 1996;245:374-93.

Maruya Y, Sasano Y, Takahashi I, Kagayama M, Mayanagi H. Expression of extracellular matrix molecules, MIMPs and TIMPs in alveolar bone, cementum and periodontal ligaments during rat tooth eruption. J Electron Microsc 2003;52:593-604.

Mazzoni A, Pashley DH, Nishitani Y, Breschi L, Mannello F, Tjäderhane $L$, et al. Reactivation of inactivated endogenous proteolytic activities in phosphoric acid-etched dentine by etch-and-rinse adhesives. Biomaterials 2006;27:4470-6.

Mazzoni A, Mannello F, Tay FR, Tonti G, Papa S, Mazzotti G, et al. Zymographic analysis and characterization of MMP-2 and -9 isoforms in human sound dentin. Journal of Dental Research 2007; $86: 436-40$

Mazzoni A, Pashley DH, Tay FR, Gobbi P, Orsini G, Ruggeri A Jr, et al. Immunohistochemical identification of MMP-2 and MMP-9 in human dentin: Correlative FEI-SEM/TEM analysis. J Biomed Mater Res A 2009:88:697-703.

Neame PJ, Kay CJ, McQuillan DJ, Beales MP, Hassell JR. Independent modulation of collagen fibrillogenesis by decorin and lumican. Cell Mol Life Sci 2000;57:859-63.

Orsini G, Ruggeri A Jr, Mazzoni A, Papa V, Mazzotti G, Di Lenarda R, et al. Immunohistochemical identification of decorin and biglycan in human dentin: a correlative field emission scanning electron microscopy/transmission electron microscopy study. Calcif Tissue Int 2007;81:39-45.

Orsini G, Ruggeri A Jr, Mazzoni A, Papa V, Piccirilli M, Falconi M, et al. Immunohistochemical identification of type I and type III collagen and chondroitin sulphate in human pre-dentine: a correlative FEI-SEM/TEM study. Int Endod J 2007; 40:669-78.

Perdigao J, Lambrechts P, Van Meerbeek B, Vanherle G, Lopes AL. Field emission SEM comparison of four postfixation drying techniques for human dentin. J Biomed Mater Res 1995;29:1111-20.

Robey PG Vertebrate mineralized matrix proteins: structure and function. Conn Tiss Res 1996; 35:131-6.

Robey PG, Fedarko NS, Hefferan TE, Bianco P, Vetter UK, Grzesik W, et al. Structure and molecular regulation of bone matrix proteins. $J$ Bone Min Res 1993;8:483-7.

Ruggeri A Jr, Prati C, Mazzoni A, Nucci C, Di Lenarda R, Mazzotti G, et al. Effects of citric acid and EDTA conditioning on exposed root dentin: An immunohistochemical analysis of collagen and proteoglycans. Arch Oral Biol 2007;52:1-8.

Sato R, Yamamoto H, Kasai K, Yamauchi M. Distribution pattern of versican, link protein and hyaluronic acid in the rat periodontal ligament during experimental tooth movement. J Periodontal Res 2002; 37:15-22.

Septier D, Hall RC, Embery G, Goldberg M. Immunoelectron microscopic visualization of pro- and secreted forms of decorin and biglycan in the predentin and during dentin formation in the rat incisor. Calcif Tissue Int 2002; 69:38-45. 
Shibata S, Kaneko S, Yanagishita M, Yamashita Y. Histochemical localization of hyaluronan and versican in the rat molar dental pulp. Arch Oral Biol 1999; 44:373-6.

Shibata S, Yoneda S, Yanagishita M, Yamashita Y .Isolation of proteoglycan (versican) aggregate from rat dental pulp. Arch Oral Biol 2000; 45:563-8.

Shibata S, Yoneda S, Yanagishita M, Yamashita Y. Developmental changes and regional differences in histochemical localization of hyaluronan and versican in postnatal molar dental pulp. Int Endod J 2002; 35:159-65.

Shinomura T, Jensen $\mathrm{KL}$, Yamagata M, Kimata K, Solursh M. The distribution of mesenchyme proteoglycan ( $P G-M)$ during wing bud outgrowth. Anat and Embryol 1990;181:227-33.

Sone S, Nakamura M, Maruya Y, Takahashi I, Mizoguchi I, Mayanagi $\mathrm{H}$, et al. Expression of versican and ADAMTS during rat tooth eruption. J Mol Histol 2005;36:281-8.

Suppa P, Ruggeri A Jr, Tay FR, Prati C, Biasotto M, Falconi M, et al. Reduced antigenicity of type I collagen and proteoglycans in sclerotic dentin. J Dent Res 2006; 85:133-7.

Teti G, Mazzotti G, Zago M, Ortolani M, Breschi L, Pelotti S, et al. HEMA down-regulates procollagen alphal type I in human gingival fibroblasts. J Biomed Mater Res A 2008;90A:256-262.

Tsubota M, Sasano Y, Takahashi I, Kagayama M, Shimauchi H. Expression of MMP-8 and MMP-13 mRNAs in rat periodontium during tooth eruption. J Dent Res 2002;81:673-8.

Waddington RJ, Hall RC, Embery G, Lloyd DM. Changing profiles of proteoglycans in the transition of predentine to dentine. Matrix Biol 2003;22:153-61.

Waddington RJ, Moseley R, Smith AJ, Sloan AJ, Embery G. Fluorideinduced changes to proteoglycan structure synthesised within the dentine-pulp complex in vitro. Biochim Biophys Acta 2004;1689: 142-51.

Warshawsky H, Moore G. A technique for the fixation and decalcification of rat incisors for electron microscopy. J Histochem Cytochem 1967; 15:542-9.

Yamauchi S, Cheng $H$, Neame $P$, Caterson B, Yamauchi M. Identification, partial characterization, and distribution of versican and link protein in bovine dental pulp. J Dent Res 1997;76:1730-6.

Yoshiba N, Yoshiba K, Iwaku M, Ozawa H. Immunolocalization of the small proteoglycan decorin in human teeth. Arch Oral Biol 1996; 41:351-7.

Zimmermann DR, Dours-Zimmermann MT, Schubert M, BrucknerTuderman L. Versican is expressed in the proliferating zone in the epidermis and in association with the elastic network of the dermis. $\mathrm{J}$ Cell Biol 1994;124:817-25.

Zimmermann DR, Ruoslahti E. Multiple domains of the large fibroblast proteoglycan, versican. EMBO J 1989;8:2975-81. 
A. Ruggeri et al. 